\title{
Intelligent Support Mechanisms in Adaptable Human-Computer Interfaces
}

\author{
D. Spath - Submitted by H. Weule (1) \\ Received on January 14,1993
}

Summary

Advanced production technology is not only characterized by higher automation of production flow and control, but more and more measured at the level of the ergonomics of man-machine interaction. Although much effort has been devoted to user friendly design and improved interface techniques, today's systems do not take into account their individual user's problems and tasks. One possible answer to this problem is the design of 'cooperative', adaptable or adaptive user interfaces. The idea proposed is to adapt interface behavior (presentation and dialog control) on account of individual user differences or user problems, by reasoning about user intensions in situational work contexts.

This paper presents the theory behind and an approach for design of adaptable/adaptive human-computer interfaces for software applications in the field of production technology, exemplified by a shop floor technology planning tool.

\section{Keywords}

Computer systems, Human factors engineering, Conceptual model 1. Foreword

\subsection{Introduction}

Increasingly exigent demands for higher productivity and enhanced quality in the field of international competition render it essential for companies to use complex and versatile information processing components. In selecting appropriate systems, the technology itself is only one of the aspects involved. The successful use of modern-day information technology depends increasingly on the userfriendliness of the computer-supported systems concerned, and their acceptance by the human staff involved.

\subsection{Motivation}

Adequate human engineering in production processes has been the subject of numerous papers in recent years, focussing predominantly on approaches for improving operability by means of appropriate design measures in the software ([Fa87]) and hardware ([Ba88]) of coimputer-supported components in those systems closely involved with the actual shopfloor manufacturing process. The results achieved here make a significant contribution to improving the ergonomics of present-day computer components.

Future systems, however, pose wider-ranging requirements for human/computer interaction. Previous approaches saw the human staff solely as operators of a technical system (e.g. a machine, a control system, etc.), so that the people involved frequently had to perform only simple, undemanding tasks. More recent approaches increasingly recognize how efficaciously human capabilities can be utilized for the production process, and therefore attempt to integrate a company's human resources more effectively into the process concerned (keyword: autonomous, independently responsible action) (cf. [Ho92], [Mi92]) (Fig. 1).
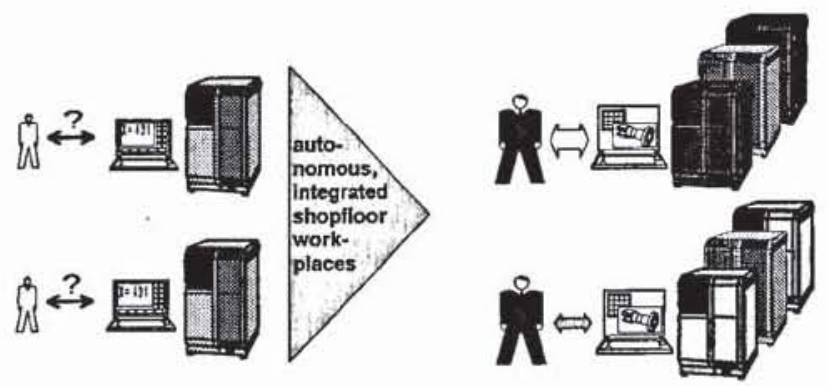

Fig. 1: How the human operator's role in the production process will change

For the human operator, this approach entails tasks of a fundamentally new nature, such as (parallel) performance of relatively sophisticated activities in integrated workplaces (planning, programming, testing, etc.). Efficacious execution of these tasks depends largely on how efficiently the human operator interacts with the machine concerned, a context in which intelligent computer support is becoming increasingly crucial. Unfortunately, inadequate attention is given to this requirement in present-day user interfaces.

\subsection{Objective}

The aim of this paper is to present an approach for intelligent support of human/computer interaction in the context of software applications for production engineering. The central ideas are explained in terms of a concrete example. The underlying theory of adaptable user interfaces is briefly outlined.

\section{Typical application}

In order to illustrate the problems involved in human/computer interaction, a typical example from a manufacturing context will be adduced here: a system used in the production engineering laboratory of Karlsruhe University for shopfloor-oriented technology planning.

The configurable technology planning system ("TechPlan"), developed at the Institute of Machine Tools and Production Science, supports knowledge-based work planning: the user is guided through the planning sequence by means of simple softkeys, with each step in the planning job (e.g. chucking tool selection, machining strategy specification, etc.) being solved by knowledge-based methods. The result of technology planning is a machine-oriented NC program for turning and milling cells (cf. also [Ev91]).

One of the planning steps, for example, is specification of the machining range. In outside turning, the user must first define the horizontal boundaries, and then the vertical ones. The interaction problem now lies in the mechanism provided for entering the boundary pairs involved in each case. Irrespective of the capabilities or working habits of a particular user, the system always "dictates" the same entry sequence and entry technique (see Fig. 2). While a novice would at first be pleased to be given more guidance for the entry procedure, practised users, with their own working methods, would be more likely to want to specify their own entry sequences.



Fig. 2: Typical example of the TechPlan technology planning system in use

\section{Approaches for user support}

Various papers in the field of user interface design over the past years ([BaSc88]) have dealt with the modalities of user support in a system interaction context.

Basic approaches have already been tried out in a number of different applications, and today form an important constituent of modern-day man/machine interfaces (Fig. 3).

These approaches, however, exhibit a variety of deficits.

The idea of standardized and/or individualized interaction mechanisms is to reduce the learning outlay by communicatory transferral of the learning successes achieved. This approach focusses primarily on the interaction problem, i.e. operation of the system, and less on the objective problem, i.e. representation of the task involved (cf. [St88]). 
The use of heip systems aims to improve a system's learnability and selfexplanatory capabilities. Here, the assistance offered is designed without reference to the user, i.e. each time a problem occurs, the assistance offered will be the same (lack of user/task referencing).

The purpose of extended dialogue mechanisms is to help to improve a system's operability by offering different forms of dialogue. But this overlooks the fact that functions of this kind are advantageous only if the user already possesses basic knowledge of how to operate the system and of the dialogue alternatives on offer.

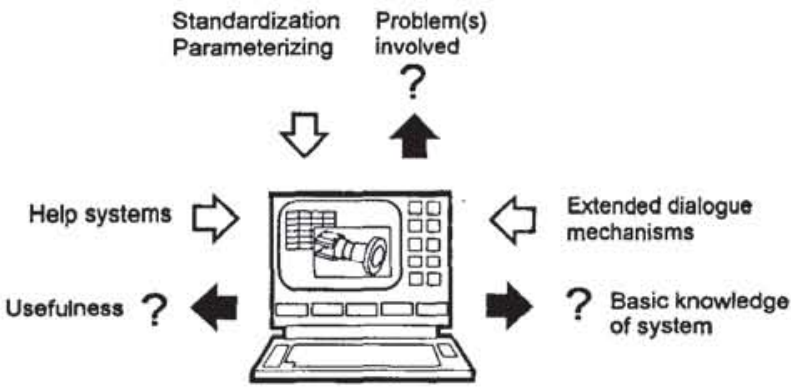

Fig. 3: Approaches to man/machine interaction support

\section{Intelligent support mechanisms}

To demonstrate user- and task-supported man/machine interaction, an adaptable user interface for the TechPlan technology planning system was developed as an example. After the requirements had been analysed, two intelligent support systems (the Dialogue Assistant and the Tutor) were designed and implemented. Figure 4 shows how the support components are integrated into the TechPlan user environment.

\subsection{Determining the requirements involved}

The requirement profile for an intelligent user support system was determined within the framework of a comprehensive analysis, in which the TechPlan technology planning system and six other of the institute's shopfloor-oriented software systems were examined. The aim here was to investigate the particular features of the user group concerned, of the field of application, and of the operating technologies employed, and then to identify the specific problem areas involved, like user qualifications, operating defects, etc. (Fig. 5).

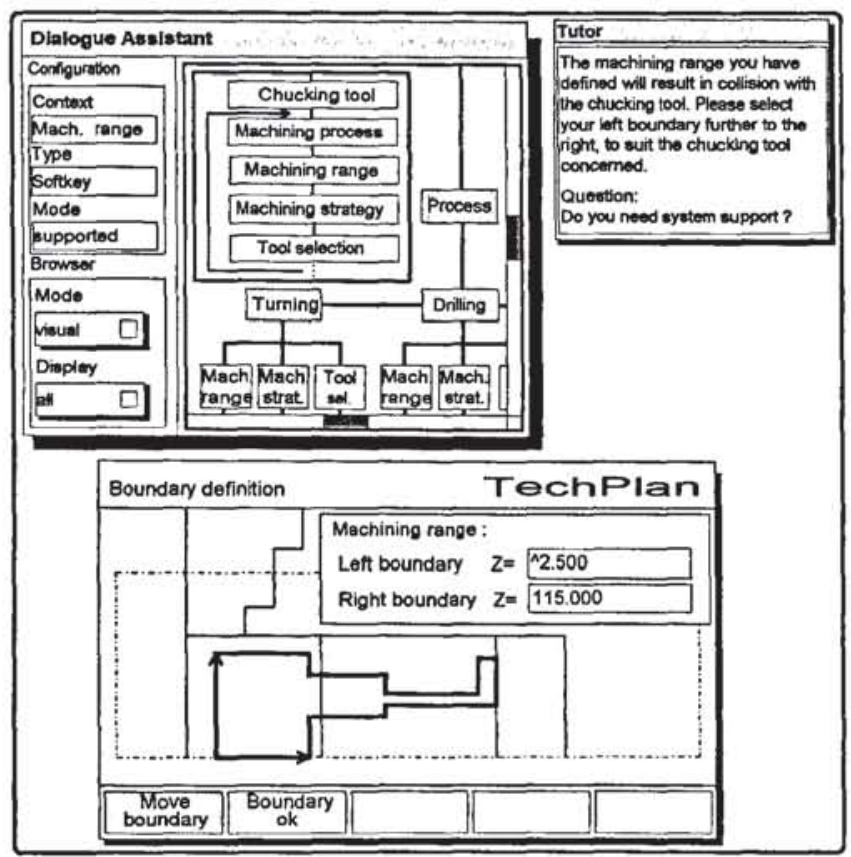

Fig. 4: The adaptable user interface of the TechPlan technology planning system

The next step was now to use the characterization of the target systems as a basis for formulating the concrete requirements which an operator-supportive user interface must meet.

Against the background of extremely heterogeneous user profiles and differing task definitions, a general demand emerges for a user-referenced, task-oriented and situationally appropriate response capability. The particularly wide variety of different user qualifications and operator capabilities in the shopfloor area entails a concomitant requirement for a more flexibly conceived dialogue facility. In order to meet the needs of an increasing number of users with low operator qualifications or even none at all, the support approach must aim to enhance the goal-directed aspects of the learning process. To increase the efficiency of the help functions in terms of better cooperation between the support system and the user, the form of support features offered must be individually tailored to the level of knowledge possessed by the actual user concerned.



Fig. 5: $\quad$ Problem areas for shopfloor-oriented computer systems

\subsection{Concept and implementation}

The requirements involved form the starting point for designing and implementing intelligent support mechanisms for an adaptable user environment. For this purpose, recourse was had to extensive previous work carried out at the institute in the field of man/machine communication (cf. [L089]), and reference was also made to various papers with similar subject-matter (cf. [GOT89] and [Ho91]).

Within the framework of a basic research project, tools for creating adaptable human/computer interfaces have been developed at the Institute of Machine Tools and Production Science (cf. [SFB92]). A distinction is made between two different levels in this context. Appropriate modelling tools have been developed to determine the adaptation requirement. Intelligent system behaviour is operationalized by adaptable support components.

\subsubsection{Support components}

Two intelligent support components have been developed. One of these is graphics-based, and serves as a visual aid, while the other component is textoriented, and possesses an informative function. Both components have been designed to be application-neutral, for adaptation to the specific needs involved in technology planning during actual use.

\section{The Tutor component}

The tutor component constitutes an information-based support component. Its job is to provide textual information for supporting the user in establishing, extending and correcting the mental model.

The tutor possesses various functions. The navigation function utilizes instructions and warnings to implement a system-side user prompting feature in the event of deviations from target. The incorporation of explanations and reasons for particular dialogue situations serves to clarify the questions involved and to establish and extend the user's mental model (explanatory function). Within the framework of the control function, an option has been implemented to enable users to execute various adaptation settings themselves. The training function has been developed with a view to expanding knowledge of the system, providing detailed explanations and comprehensive examples enabling users to comprehend the intra-system constraints and the specialized causal relationships involved.

In addition, graduated tutor responses provide an option for imparting the information on offer to the user in appropriate steps. The advantage of this method is that it avoids repeated presentation of the same tutor information in the same context, and increases user attention over a lengthy period.

Figure 6 shows the specification of the tutor component, and a typical tutor response after the boundaries of a machining range have been incorrectly defined. 


\begin{tabular}{|c|c|}
\hline 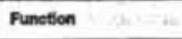 & Desetiption \\
\hline Nangation & $\begin{array}{l}\text { - eyctem-ide guidance } \\
\text { - correction of orrors }\end{array}$ \\
\hline Training & 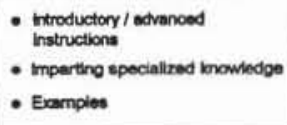 \\
\hline Contral & - user-sido adapton \\
\hline Expentedion & 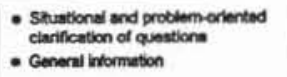 \\
\hline
\end{tabular}

\begin{tabular}{|l|l|}
\hline Tutor component \\
\hline Mode adaptive & $\begin{array}{l}\text { The machining range you have defined will } \\
\text { result in collision with the chucking tool. } \\
\text { Please select your left boundary further to } \\
\text { the right, to suit the chucking tool concemed. }\end{array}$ \\
\begin{tabular}{|l|l|}
\hline Context \\
Mach. range \\
Class
\end{tabular} & $\begin{array}{l}\text { Hint/Question } \\
\text { Parameter }\end{array}$ \\
\hline Collision \\
\hline
\end{tabular}

Fig. 6: Specification and user interface of the tutor component

\section{The Dialogue Assistant component}

The purpose of the Dialogue Assistant component is to implement behavioural adaptation of the user interface: this component provides two different basic functions, the dialogue configuration and the dialogue browser functions.

Within the framework of the dialogue configuration function, the Dialogue Assistant component is designed to render the system's operator control behaviour more flexible, with dynamic variation of interactive prompting ensuring user-tailored responses.

The dialogue browser function incorporates a graphical visualization tool for depicting and controlling dialogue sequences. The requirement for a tool of this kind is entailed by the frequently high complexity of dialogue structures (long dialogue paths, large number of dialogue contexts) in shopfloor applications.

\begin{tabular}{|c|c|}
\hline 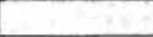 & Dlalogue Configuration \\
\hline \multicolumn{2}{|c|}{ IS Task: Diwogue control adeption } \\
\hline Aspecta: & $\begin{array}{l}\text { - User refarenco } \\
\text { - Situation reference } \\
\text { - Probiem orientation }\end{array}$ \\
\hline \multicolumn{2}{|c|}{$\begin{array}{l}\Rightarrow \text { Eftect rendering the operutor control behaviour } \\
\text { more flenibio }\end{array}$} \\
\hline & Dlalogue Browser \\
\hline \multicolumn{2}{|c|}{ CS Task: Graphical visualization of the dialogue structure } \\
\hline Aspects: & $\begin{array}{l}\text { - Orientation support } \\
\text { - Visual explanebion / training } \\
\text { Oirect dialogue accose }\end{array}$ \\
\hline$\Rightarrow$ Eneot: onh & Ianced efficiency in dialogue handing \\
\hline
\end{tabular}

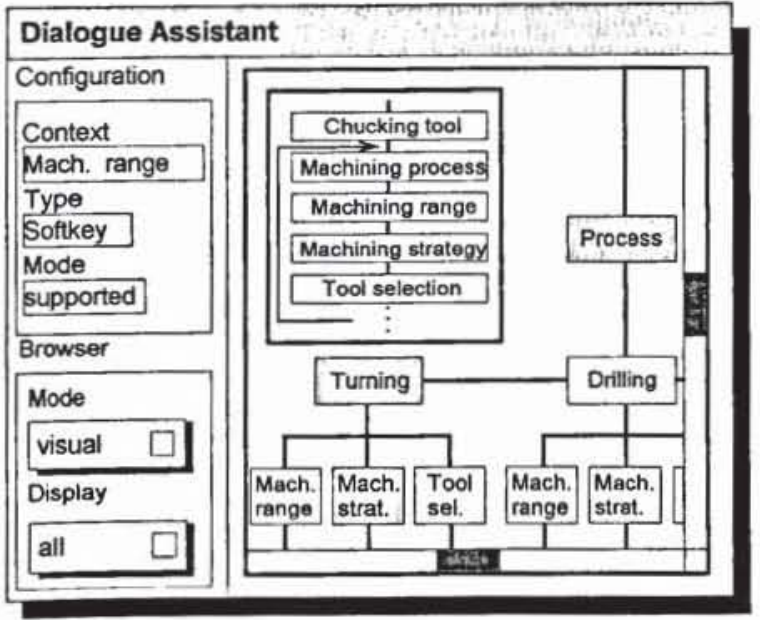

Fig. 7: Specification and user interface of the Dialogue Assistant component
The option for graphical depiction of differing dialogue structures represents an efficient enhancement of the tutor component (in connection with the training function, for example).

Figure 7 shows the specification of the Dialogue Assistant component, and an example of system-supported interactive prompting for selecting the machining strategy.

The Tutor and the Dialogue Assistant have been implemented in the form of open software components in the $\mathrm{C}$ programming language, utilizing sophisticated software development tools. The TechPlan application presented in this paper is currently running on a DECstation 5000 under the Ultrix operating system. Further developments of the system aim at porting onto a shopfloor-oriented system, preferably featuring control of the turning/milling cell itself

\subsubsection{Modelling tools}

Intelligent use of the support systems presented above in software applications requires a wider-ranging view of the user interface. In this context, an adaptable user interface provides the requisite foundations (cf. [L089], [BTN90] and [NoSt89])

The essential characteristic of an adaptable user interface is the ability to adjust to the individual needs of different users. Depending on the previous knowledge, the experience and the skills of the user concerned, and taking into account his or her goals and purposes, it is able to alter their appearance and their behaviour to suit the user and the task involved.

A distinction can be drawn between two basic variants of adaptability in adaptable user interfaces, depending on the source used for triggering an adaptation: if the user is the initiator, this is referred to as adapted adjustment, while a system-side adaptive intervention is called adaptive adjustment.

The adaptive system adjustment facility, in particular, is based on intra-system modelling of characteristic information on the user and his or her tasks. The associated models are referred to as the user model and the task model.

During a session, user inputs and system outputs are logged and filed in the appropriate components (user modelling component and task modelling component). Utilizing a strategic decision-making component (strategy component), the support requirement is determined and the adapted system responses are triggered (cf. [Zi91]) (Fig. 8).

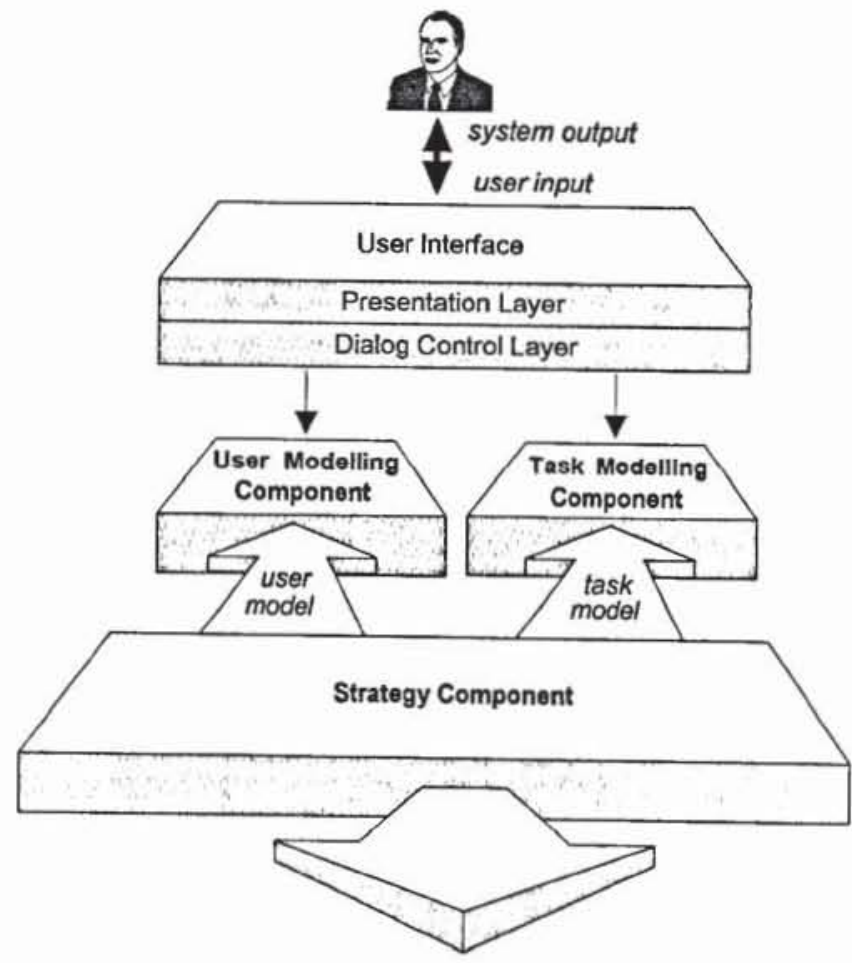

adaptable system control

\section{Fig. 8: Principle behind an adaptable user interface}

The idea of the user modelling component is to describe the user in terms of his or her interactions with the system. For this purpose, information obtained from the dialogue, i.e. user inputs and system outputs, are stored in memory (interaction $\log$ ) and evaluated against predefined criteria. The aim of maximizing the precision of user characterization in the environment of the application 
involved can be implemented, for example, by classification into stereotypes (novice, advanced user, expert, etc.) (cf. also [KoWa89])

The purpose of the task modelling component is to describe the user's tasks, wishes and problems in coping with the job involved. For this purpose, utilizing internal representation formalisms, referred to as action plans (e.g. [Ma86]), user interactions are analysed during the session (action plan tracking) and checked for permissibility or goal-directedness. By deriving hypotheses on the user's goals, intentions and problems (e.g. "Tool not suitable for selected machining job"), clues are obtained enabling conclusions to be drawn on the causes involved (e.g. "Mental model of the tool/machining process relationship is incomplete").

The strategy component implements a framework for describing strategic decision-making knowledge in selecting adapted system behaviour. Using a logicbased system of rules, the user model and the task model are dynamically evaluated, and the degree and nature of the support requirement determined. This results in activation of memory-stored intelligent support mechanisms designed to provide the appropriate form of assistance.

Implementation of the adaptable user interface was based on an extended architecture for user interfaces Figure 9 shows the structure of the system components and the data flows for information exchange.

\subsection{Empirical finding}

The support components developed have been tested and evaluated within the framework of a field trial in the Manufacturing Engineering Laboratory: test persons with differing knowledge of the system (novices, users with brief training, advanced users and practised users) were asked for their reactions after working with the intelligent support mechanisms.

The test candidates gave a generally favourable rating to the adaptable user interface. The Tutor component's ability to offer problem-oriented explanations and instructions was perceived as very helpful. The idea of dynamizing the dialogue sequence, originally greeted with scepticism, was generally accepted by the test persons involved.

Notable features included keen interest (particularly from the beginners) in learning how they personally had been graded in the user model, and the resulting motivation to improve their performance. There was, too, adequate discussion of the user data stored in memory, and coding procedures were tested for a real-use scenario.

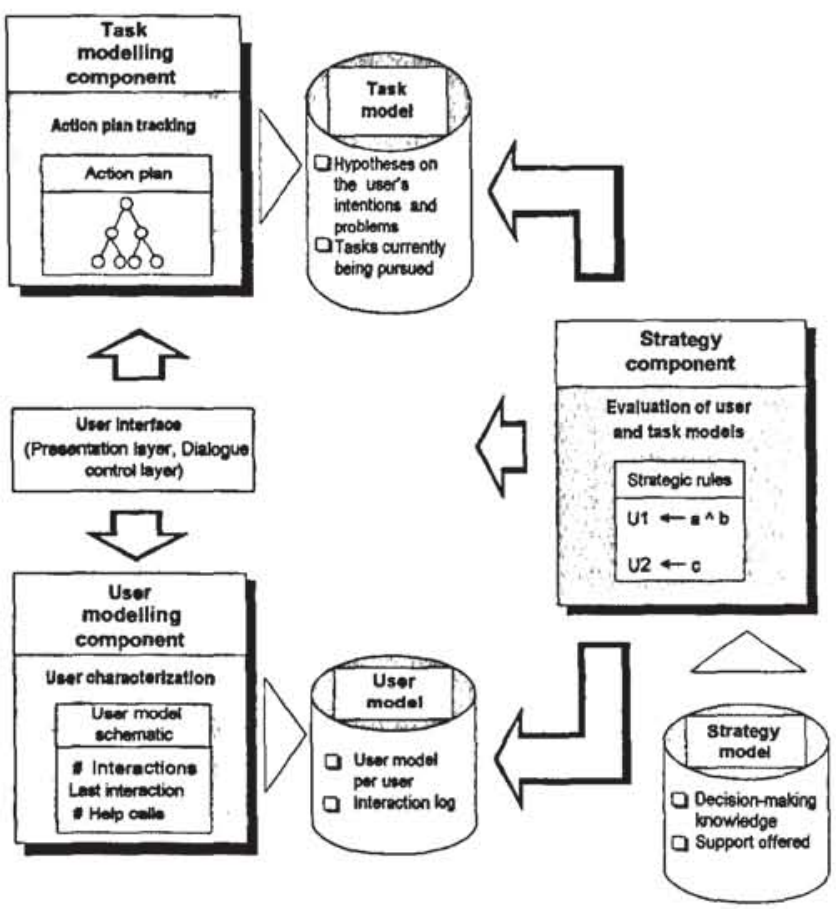

Fig. 9: Architecture of an adaptable user interface

\section{Conclusion}

The aim of this paper was to present an approach for improving man/machine interaction by means of intelligent usage mechanisms. For this purpose, an adaptable user interface was developed, capable of implementing adaptive system behaviour on the basis of user and task models. The most significant performance features of the support mechanisms developed were efficient support of the learning process and optimization of the operator control behaviour by means of user-adapted dialogue control. The field of application for the system described lies in the area of technology planning, which must be seen as a subsystem within a sophisticated, integrated shopfloor workplace.

The approach presented opens up a broad spectrum of possible applications. Against the background of more closely integrated production areas, use in the field of groupwork-supportive $\mathrm{man} / \mathrm{machine}$ and $\mathrm{man} / \mathrm{man}$ communication would be a very promising approach. In this context, adapted forms of dialogue could help to improve problem-related communication between different departments, and intelligent coordinatory and control functions could provide optimizing support for work in distributed teams.

\section{References}

[Ba88] Balzert, H. E/A-Geräte für die Mensch-Computer-Interaktion. In Balzert, H. (Hrsg.) Einfuihrung in die Software-Ergonomie. W. de Guyter, Berlin, 1988.

[BaSc88] Bauer, J., Schwab, T. Anforderungen an Hilfesysteme. In Balzert, H. (Hrsg.) Eimfihrung in die Software-Ergonomie. W. de Gruyter, Berlin, 1988.

[BTN90] Browne, D., Totterdell, P. Norman, M. Adaptive User Interfaces. Academic Press, London, 1990.

[Ev91] Eversheim, W., Marczinski, G., Cremer, R. Structered Modelling of mamufacturing processes as NC-Data preparation. In Annals of the CIRP, Vol. 40, 1, 1991.

[Fa87] Fähnrich, K.P. (Hrsg.) Software-Ergonomie, State of the Art S. Oldenburg Verlag, München, 1987

[GOT91] Grunst, G., Oppermann, R., Thomas, C. (Hrsg.) Intelligente Bemutzerschnittstellen. In Benutzerschnittstelle (Theorie und Praxis der Wirtschaftsinformatik). Forkel, 28 (1991)160, S. 35 - 47.

[Ho91] Hoschka, P. Assisting Computer - A new Generation of Support Systems. In Proc. 4. Internationaler GI-Kongre $B$ Wissensbasierte Systeme - Künstliche Intelligenz am 23.-24.10.1991 in München. Springer, Berlin, 1991

[Ho92] Holz, B Die Kraftreserven in der Fabrik stecken in der Fertigung. VDI-Nachrichten, 3, 1992, S. 13.

[KoWa89] A. Kobsa, W. Wahlster User Models in Dialog Systems. Springer. Berlin, 1989.

[L889] Loffler, L. Adaptierbare und adaptive Bemutzerschnittstellen. Diss. Universität Karlsruhe, Karlsruhe, 1989.

[Ma86] Mac an Airchinnigh, M. Conceptsal Models an Command Languages. In Hopper, K., Newman, I.A. (Hrsg.) Foundation for HumanComputer Communication. Proc. of the IFIP WG 2.6 Working Conference on The Future of Command Languages, Rome, Italy, North-Holland, Amsterdam, 1986, S. 99-124.

[Mi92] Milberg, J. Flexibilität braucht dezentrale Organisation. VDINachrichten, 1/2, 1992, S. 11

[NoSt89] Norcio, A.F., Stanley, J. Adaptive Human-Computer Interfaces: A Literatur Survey and Perspective. IEEE Transactions on Systems, Man and Cybernetics 19 (1989), 2, 399-408.

[SFB92] Universităt Karlsnuhe Rechnerintegrierte Konstruktion und Fertigung von Bauteilen. Arbeits- und Ergebnisbericht des Teilprojekts A4.2 im Sonderforschungsbereich 346, Karisruhe, zu erscheinen im Frühjahr 1993.

[St88] Streitz, A. in Baizert, H. (Hrsg.) Einfuhrung in die Software-Ergonomie. W. de Gruyter, Berlin, 1988.

[Zi91] Zimek, S. Design of an Adaptable/Adaptive User Interface Mana gement System in Production. In Bullinger, H.-J. (Hrsg.) Human Aspects in Computing. Tagungsband der $\mathrm{HCI}$ International Conference ' 91 in Stuttgart, Elsevier Publisher, 1991. 